\title{
Intertwined vestigial order in quantum materials: nematicity and beyond
}

\author{
Rafael M. Fernandes, ${ }^{1}$ Peter P. Orth ${ }^{2}$ and Jörg Schmalian ${ }^{3}$ \\ ${ }^{1}$ School of Physics and Astronomy, University of Minnesota, Minneapolis, Minnesota 55455, USA \\ ${ }^{2}$ Department of Physics and Astronomy, Iowa State University, Ames, Iowa 50010, USA \\ ${ }^{3}$ Institute for Theory of Condensed Matter and Institute for Solid State Physics, \\ Karlsruhe Institute of Technology (KIT), Karlsruhe, Germany
}

A hallmark of the phase diagrams of quantum materials is the existence of multiple electronic ordered states, which, in many cases, are not independent competing phases, but instead display a complex intertwinement. In this review, we focus on a particular realization of intertwined orders: a primary phase characterized by a multi-component order parameter and a fluctuation-driven vestigial phase characterized by a composite order parameter. This concept has been widely employed to elucidate nematicity in iron-based and cuprate superconductors. Here we present a group-theoretical framework that extends this notion to a variety of phases, providing a classification of vestigial orders of unconventional superconductors and density-waves. Electronic states with scalar and vector chiral order, spin-nematic order, Ising-nematic order, time-reversal symmetry-breaking order, and algebraic vestigial order emerge from one underlying principle. The formalism provides a framework to understand the complexity of quantum materials based on symmetry, largely without resorting to microscopic models. 


\section{INTRODUCTION}

Many quantum materials are characterized by a rich phase diagram, in which numerous order parameters assume finite values in neighboring regions of the parameter space of temperature, chemical composition, mechanical strain, and electromagnetic fields. The natural instinct one has to capture this physics is to assign the different phases to competing order parameters. For example, if one finds antiferromagnetism (with order parameter m) and superconductivity (with order parameter $\Delta$ ) nearby, one writes individual Ginzburg-Landau expansions for the free energy $f_{m}$ and $f_{\Delta}$ for both degrees of freedoms, coupled by a symmetry-allowed term such as $f_{m-\Delta}=\gamma \mathbf{m} \cdot \mathbf{m}|\Delta|^{2}$. Positive $\gamma$ amounts to phase competition while negative $\gamma$ causes one phase to attract the other. While this approach proved to be very efficient in many cases [1], Landau theory cannot explain why multiple phases emerge close to each other in a phase diagram. Addressing this question usually requires a microscopic description in terms of a model Hamiltonian, a task that can be technically challenging. Given the abundance of complex phase diagrams in correlated electronic systems, it is desirable to identify general principles to describe the close relationship between their multiple ordered states.

An underlying general principle to rationalize complex phase diagrams without necessarily resorting to a microscopic description was recently advocated in Ref. [4] and is generally referred to as intertwined order. The idea is that multiple phases of a rich phase diagram are born out of a primary state. A prime example for such a behavior is that of pair-density-wave order, which entangles superconductivity and density waves [5] 10]. Intertwined orders can also arise due to the interactions induced by a primary order parameter near a quantum phase transition. For example, antiferromagnetic or nematic fluctuations near quantum critical points have been proposed to provide or enhance the pairing interactions for a superconducting phase [11, 12].

In this review, we focus on a particular realization of intertwined phases in terms of vestigial - or composite order. Composite order exists when higher order combinations of potentially symmetry-breaking order parameters condense. Consider a complex multi-component field $\eta_{\alpha}$, where $\alpha$ labels the order parameter components. A finite expectation value $\left\langle\eta_{\alpha}\right\rangle$ would break a certain symmetry of the system - for instance, time-reversal in the case of ferromagnetism or translational symmetry in the case of charge order. Composite order then corresponds to the case where certain combinations of the product of the order parameters are on average non-zero, whereas each individual order parameter remains zero on average:

$$
\left\langle\eta_{\alpha}^{*} \eta_{\beta}\right\rangle \neq 0 \quad \text { but } \quad\left\langle\eta_{\alpha}\right\rangle=0
$$

The bilinear combination $\left\langle\eta_{\alpha}^{*} \eta_{\beta}\right\rangle$ behaves itself as an order parameter, which breaks only a subset of the symmetries broken by $\eta_{\alpha}$. For this reason, the composite order is called a vestige of the primary phase where $\left\langle\eta_{\alpha}\right\rangle$ is finite. This makes both the composite and primary orders naturally intertwined. At first glance, this scenario may seem rather contrived. However, as we will show here, it naturally arises in many quantum materials, when the primary order parameter has multiple components, such that the primary phase is degenerate. In Eq. (1) we allowed for $\eta_{\alpha}$ to be complex. This is relevant for superconductors or incommensurate density-wave states.

There are two complementary ways to approach a composite ordered phase. If one starts from the primary ordered phase, composite order can be understood as a partial melting of the former, before the system goes to a completely disordered phase [13, 14. Conversely, starting from the disordered phase, vestigial order can be understood as a fluctuation-induced composite order, i.e. a state of symmetry-breaking fluctuations [15. Since these fluctuations are naturally strong near the phase transition of the primary order parameter, this line of reasoning explains the existence of multiple nearby ordered states, largely using symmetry arguments. It allows for predictability of complex phase diagrams, even in strongly correlated materials.

The richness of the phase diagrams involving vestigial orders contrast with the well-known phase diagrams involving competing phases [16, 17. In the latter, the system displays either a bicritical or a tetracritical point, depending on whether the competing orders phase-separate or coexist, respectively. In contrast, several outcomes are possible in the former case, some of which are illustrated in Fig. 1. A key feature is that the behavior at finite temperatures can be very different than that at $T=0$. For instance, in the simple case of split vestigial and primary transitions at finite temperatures, the system may display two quantum critical points (Fig. 1 a), a single first-order quantum phase transition (Fig. 1b), or even a single quantum critical point (Fig. 17). Importantly, in several models more than one vestigial order appears. Two vestigial orders can appear if the non-zero component of the primary order parameter $\left\langle\eta_{\alpha}\right\rangle$ changes along the phase diagram (Fig. 11d). Moreover, certain systems can display additional vestigial phases formed by composite trilinear order parameters (Fig. 17) or quasi-long-range ordered bilinears (Fig. 1f). Examples of these cases will be given throughout the review.

Historically, fluctuation-induced composite order has played an important role in the area of frustrated magnetism and is closely related to the concept of order-from-disorder [18 20. The identification of an emergent, vestigial Ising degree of freedom in a frustrated two-dimensional Heisenberg model in Ref. [21] is a beautiful and influential example 


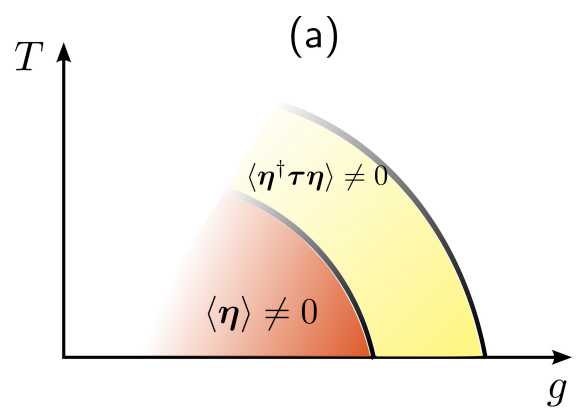

(b)
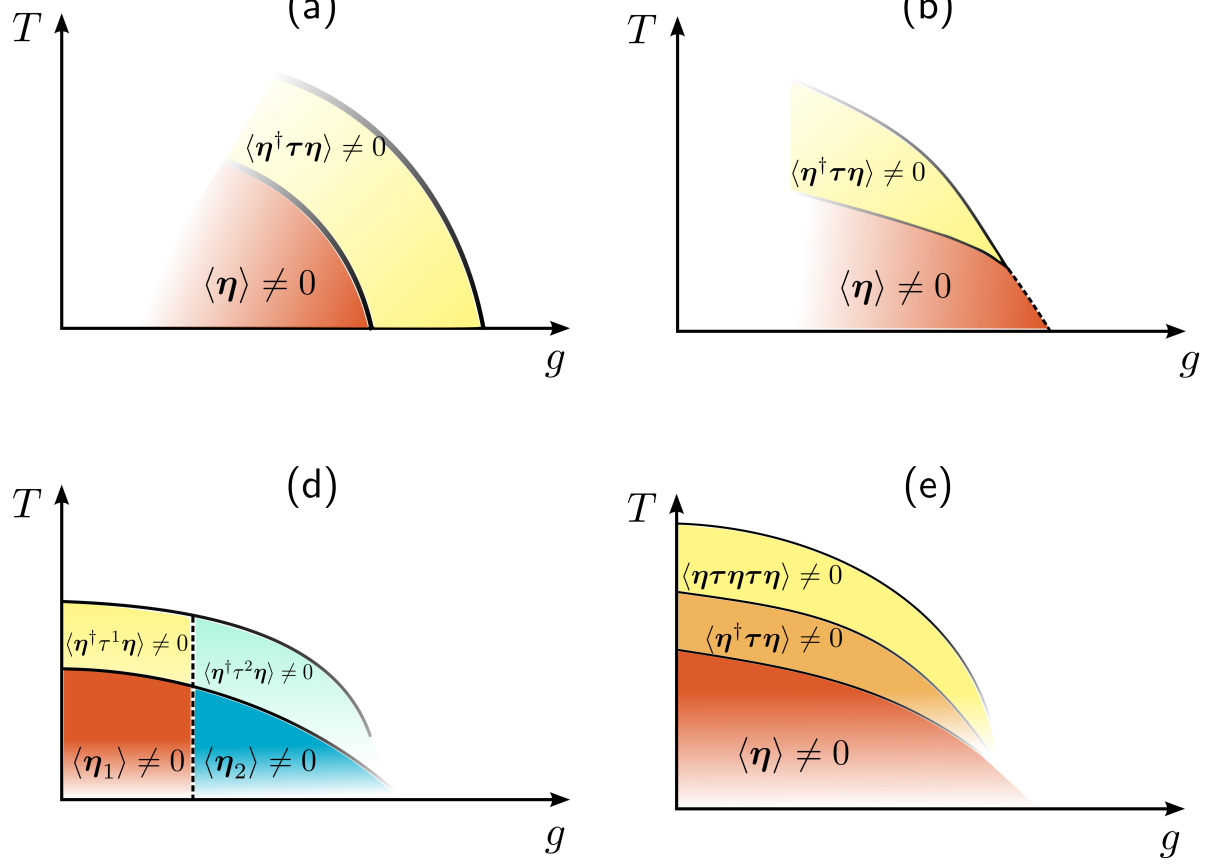

$(\mathrm{e})$

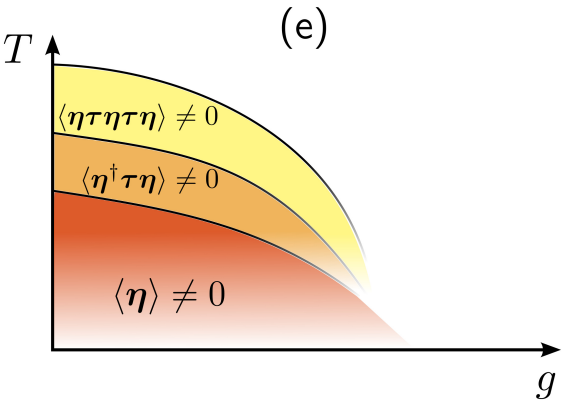

(c)

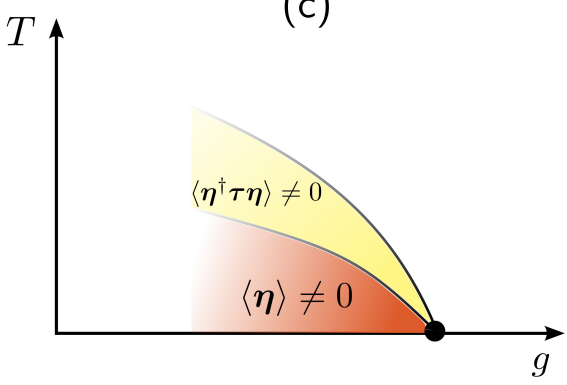

FIG. 1. Schematic phases diagrams for the primary order (denoted by the parent order parameter $\langle\boldsymbol{\eta}\rangle$ ) and the vestigial order (denoted by the composite order parameters $\left\langle\boldsymbol{\eta}^{\dagger} \boldsymbol{\tau} \boldsymbol{\eta}\right\rangle$ and $\langle\boldsymbol{\eta} \boldsymbol{\tau} \boldsymbol{\eta} \boldsymbol{\eta}\rangle$ ). Second-order (first-order) transitions are denoted by solid (dashed) lines. Panels (a), (b), and (c) show three possible outcomes for the quantum phase transitions of the vesitigial and primary orders, in the case when their finite-temperature phase transitions are split. Panel (d) illustrates the appearance of two different vestigial orders when the condensed component of the order parameter $\langle\boldsymbol{\eta}\rangle$ of the primary phase changes across the phase diagram. Panel (e) displays a situation in which two different vestigial orders appear, corresponding to bilinear and trilinear composites. Panel (f) illustrates the case in which the vestigial order itself has a regime with quasi-long-range order, giving rise to a critical vestigial phase. The parameter $g$ here corresponds to some external tuning parameter. Other phase diagrams not shown here are also possible .

for vestigial order. More recently, the concept of composite order played a prominent role in the explanation of nematicity, i.e. electronically-driven rotational symmetry-breaking, in iron-based superconductors [15, 22, 25]. As we argue here, the applicability of this concept goes well beyond frustrated magnetism and nematicity, opening interesting routes to investigate unusual electronic states in unconventional superconductors and density-wave systems.

In order to move beyond particular examples and systems, it is important to put the concept of composite order on formal grounds, which can be achieved using symmetry arguments. Let the complex primary order parameter $\eta_{\alpha}$ transform under a specific irreducible representation $\Gamma$ of the symmetry group $\mathcal{G}$ of the problem. Then the components $\alpha=1, \cdots, d_{\Gamma}$ refer to the elements within the irreducible representation of dimension $d_{\Gamma}$. The composite order parameter

$$
\phi_{m}=\sum_{\alpha \beta} \eta_{\alpha}^{*} \Lambda_{\alpha \beta}^{m} \eta_{\beta}
$$

transforms under one of the irreducible representations $\Gamma^{m}$ that is contained in the product $\Gamma^{*} \otimes \Gamma[26,27$. Here $\Lambda_{\alpha \beta}^{m}$ is a $d_{\Gamma} \times d_{\Gamma^{-}}$dimensional matrix that transforms under $\Gamma^{m}$. Elementary group theoretical arguments show that symmetry-breaking composites can only be formed out of multi-component primary order parameters, i.e. $d_{\Gamma}>1$. Otherwise, $\phi_{m}$ must transform under the trivial representation and will not break a symmetry. Thus, composite order of the type Eq.22 requires a non-Abelian symmetry group $\mathcal{G}$. Fortunately, there appears plenty of those in generic condensed-matter systems.

In the remainder of this review, we will apply and generalize such symmetry arguments to analyze composite order that is driven by strong fluctuations. To set the stage, we start by discussing the case of $p$-wave unconventional superconductivity (Sec. II), followed by the cases of density-waves on the square lattice (Sec. III) and on the hexagonal lattice (Sec. IV). The latter have important consequences for the phase diagrams of iron-based superconductors and graphene, respectively. Section $\mathrm{V}$ discusses other examples and possible extensions of these ideas, including an example of a system where an emergent symmetry of the ground-state leads to the absence of vestigial order. We will demonstrate that a rich plethora of electronic states with scalar and vector chiral order, spin-nematic order, 
Ising-nematic order, time-reversal symmetry-breaking order, and critical phases emerge out of this simple underlying principle.

\section{VESTIGIAL ORDER FROM UNCONVENTIONAL SUPERCONDUCTIVITY}

To set the stage for the next sections, we start by investigating vestigial order in unconventional superconductors. As a specific example, we consider a $p$-wave superconductor on a tetragonal $(d=3)$ or square $(d=2)$ lattice. The

amplitude $\left\langle c_{\mathbf{k} \alpha}^{\dagger} c_{-\mathbf{k} \beta}^{\dagger}\right\rangle$ of a Cooper pair that consists of one electron with momentum $\mathbf{k}$ and spin $\alpha$ and another electron with $-\mathbf{k}$ and $\beta$ is efficiently characterized in terms of the $\mathrm{d}$-vector $\mathbf{d}_{\mathbf{k}}$ :

$$
\Delta_{\alpha \beta}(\mathbf{k})=\left[\left(\mathbf{d}_{\mathbf{k}} \cdot \boldsymbol{\sigma}\right) i \sigma_{y}\right]_{\alpha \beta} .
$$

Here, $\sigma_{j}$ are Pauli matrices. The Pauli principle dictates that the d-vector is odd under inversion, i.e. $\mathbf{d}_{-\mathbf{k}}=-\mathbf{d}_{\mathbf{k}}$, such that the gap function is antisymmetric with respect to the exchange of the two electrons that form the Cooper pair. In the case of a triplet Cooper pair with $S_{z}=0$, the d-vector is parallel to the $z$-axis and can be parametrized as:

$$
\mathbf{d}_{\mathbf{k}}=\hat{\mathbf{z}}\left(\eta_{x} \sin k_{x} a+\eta_{y} \sin k_{y} a\right) .
$$

Here, $a$ is the lattice constant in the $x y$ plane. The two complex order parameters $\eta_{x}$ and $\eta_{y}$ thus correspond to $p_{x}$ and $p_{y}$ superconducting states, respectively. Theoretically, $p$-wave superconductivity is expected when pairing is mediated by the exchange of ferromagnetic fluctuations. Experimentally, material candidates for $p$-wave superconductors include the ruthenate $\mathrm{Sr}_{2} \mathrm{RuO}_{4}\left[28\right.$, 29] and the doped topological insulator $\mathrm{Cu}_{x} \mathrm{Bi}_{2} \mathrm{Se}_{3}$ [30, 31].

\section{A. Symmetry classification}

We can build a Ginzburg-Landau expansion of the free energy $f$ in terms of the two-component order parameter $\boldsymbol{\eta} \equiv\left(\eta_{x}, \eta_{y}\right)$. The usual form for the expansion for a system with spin-orbit coupling and tetragonal point group $D_{4 h}$ is 32 (gradient terms are neglected for the sake of clarity):

$$
f=\frac{r}{2}\left(\left|\eta_{x}\right|^{2}+\left|\eta_{y}\right|^{2}\right)+\frac{u}{4}\left(\left|\eta_{x}\right|^{4}+\left|\eta_{y}\right|^{4}\right)+\frac{g}{2}\left|\eta_{x}\right|^{2}\left|\eta_{y}\right|^{2}+\frac{w}{8}\left(\eta_{x} \eta_{y}^{*}+\eta_{y} \eta_{x}^{*}\right)^{2}
$$

The terms that determine the allowed ground states are the quartic ones. For our purposes, it is therefore convenient to write $f$ in terms of bilinears:

$$
f=\frac{r}{2} \phi_{0}+\frac{(u+g)}{8} \phi_{0}^{2}+\frac{(u-g)}{8} \phi_{3}^{2}+\frac{w}{8} \phi_{1}^{2}
$$

where

$$
\phi_{m}=\sum_{\alpha \beta} \eta_{\alpha}^{*} \tau_{\alpha \beta}^{m} \eta_{\beta}
$$

are the possible bilinear forms, with the Pauli matrices $\tau_{\alpha \beta}^{m}$ playing the role of the matrices $\Lambda_{\alpha \beta}^{m}$ in Eq. 2 . The absence of the $\phi_{2}^{2}$ term is a consequence of the Fierz identity, $\phi_{1}^{2}+\phi_{3}^{2}=\phi_{0}^{2}-\phi_{2}^{2}$, which implies that one can always express one of the allowed bilinear forms in terms of the others. The different possible $p$-wave superconducting states are obtained by minimizing the free energy, and are given by

$$
\begin{aligned}
& \boldsymbol{\eta}_{B_{1 g}} \propto(1,0) \text { or }(0,1), \\
& \boldsymbol{\eta}_{B_{2 g}} \propto(1, \pm 1), \\
& \boldsymbol{\eta}_{A_{2 g}} \propto(1, \pm i) .
\end{aligned}
$$



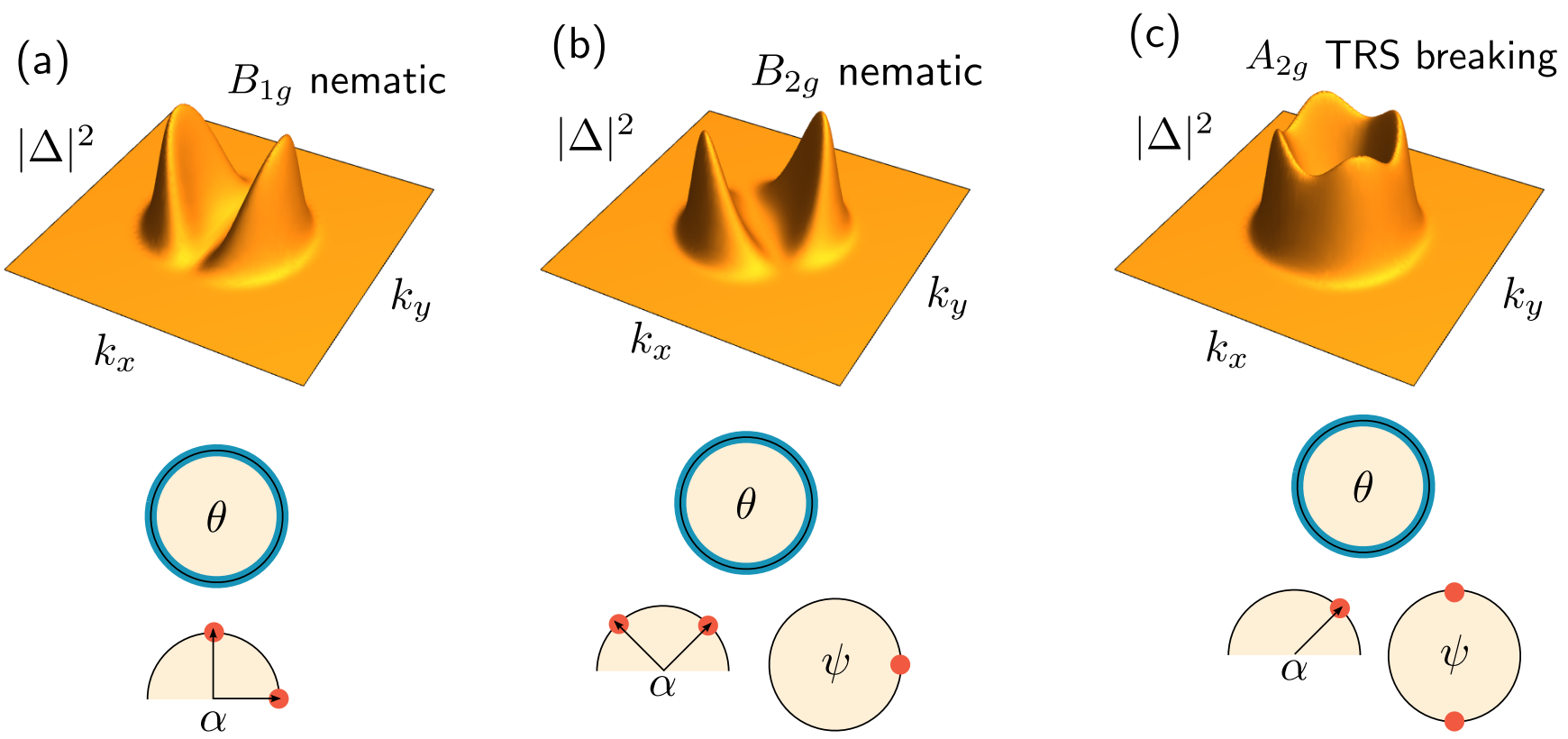

FIG. 2. The three possible $S_{z}=0$ triplet superconducting states in a tetragonal system (upper panels), and the corresponding vestigial phases (lower panels). Panel (a) shows the $B_{1 g}$-nematic state; panel (b), the $B_{2 g}$-nematic state; and panel (c), the $A_{2 g}$ time-reversal symmetry-breaking state. The primary phases are illustrated by three-dimensional plots of the gap function $|\Delta|^{2}$, defined in Eq. (4), around a circular Fermi surface. The vestigial phases are illustrated by the behavior of the phases defined in Eq. 10. While the global phase $\theta$ is always fluctuating in the vestigial phases, one of the phases $\alpha$ or $\psi$ can acquire two different values, evidencing the Ising-like character of the vestigial order parameters.

Which state is realized depends on the values of the quartic coefficients. The $B_{1 g}$ superconducting state is the ground state when $u-g<\min (0, w)$; the $B_{2 g}$ ground state takes place when $u-g>w$ and $w<0$; and the $A_{2 g}$ ground state is realized when $u-g>0$ and $w>0$.

At the superconducting transition, the global $U(1)$ symmetry is broken or, in the case of a two-dimensional system, algebraic order sets in via a Berezinskii-Kosterlitz-Thouless (BKT) transition. In addition, each of the three possible ground states also breaks a discrete Ising-like $\left(Z_{2}\right)$ symmetry of the system, as illustrated schematically in Fig. 2 . The $B_{1 g}$ state breaks the tetragonal symmetry of the lattice such that the $x$ and $y$ directions are inequivalent. This is shown in the upper panel of Fig. $2 a$, which plots $|\Delta|^{2}$ for a circular Fermi surface. It therefore corresponds to a $B_{1 g}$ nematic superconductor, since the system retains horizontal and vertical mirror symmetries. The $B_{2 g}$ state with $\eta_{x}= \pm \eta_{y}$ also breaks the tetragonal symmetry of the lattice, but by making the diagonal directions $x y$ and $x \bar{y}$ inequivalent (upper panel of Fig. 2p). As a result, it is a $B_{2 g}$ nematic superconductor, where the diagonal mirror symmetries are preserved. Finally, the state with $\eta_{x}= \pm i \eta_{y}$ breaks time-reversal symmetry (upper panel of Fig. 20). It supports orbital currents associated with the chirality of the gap function, and as such it transforms as the $A_{2 g}$ irreducible representation of the tetragonal group.

These properties of a $p$-wave superconductor are efficiently captured within the framework outlined in the introduction. The symmetry group of the problem is $\mathcal{G}=D_{4 h} \otimes U(1)$, where $D_{4 h}$ refers to the tetragonal point group that describes the square and tetragonal lattices, and $U(1)$ is the continuous group related to the complex nature of the order parameters $\eta_{\alpha}$. Importantly, the $\eta_{x}$ and $\eta_{y}$ order parameters transform according to the two-dimensional irreducible representation $E_{u}$ of $D_{4 h}$, i.e. $\Gamma=E_{u} \times e^{i m \theta}$. We focus on composite order parameters that do not break the $U(1)$ order parameter. The product

$$
\Gamma^{*} \otimes \Gamma=E_{u} \otimes E_{u}=A_{1 g} \oplus B_{1 g} \oplus B_{2 g} \oplus A_{2 g}
$$

is then decomposed in terms of 4 one-dimensional irreducible representations of the $D_{4 h}$ group. From Eq. (9), we conclude that there are four different values of the irreducible representation index, $m=0,1,2,3$. The associated bilinear forms are the same as those introduced in Eq. (7). In explicit form, we have: $\phi_{0}=\boldsymbol{\eta}^{\dagger} \tau^{0} \boldsymbol{\eta}=\left|\eta_{x}\right|^{2}+\left|\eta_{y}\right|^{2}$, $\phi_{1}=\boldsymbol{\eta}^{\dagger} \tau^{1} \boldsymbol{\eta}=\eta_{x} \eta_{y}^{*}+\eta_{y} \eta_{x}^{*}, \phi_{2}=\boldsymbol{\eta}^{\dagger} \tau^{2} \boldsymbol{\eta}=i\left(\eta_{x} \eta_{y}^{*}-\eta_{y} \eta_{x}^{*}\right)$, and $\phi_{3}=\boldsymbol{\eta}^{\dagger} \tau^{3} \boldsymbol{\eta}=\left|\eta_{x}\right|^{2}-\left|\eta_{y}\right|^{2}$. Because $\phi_{0}$ transforms as the trivial irreducible representation $A_{1 g}$, it does not break any symmetry of the system. As a result, it cannot serve as a vestigial order parameter, but instead corresponds to fluctuations present in the vicinities of the normal-state to superconducting phase transition, regardless of the nature of the superconducting state. $\phi_{1}$, on the other hand, 
transforms as the $B_{2 g}$ irreducible representation and, as such, is a nematic vestigial order parameter that breaks the tetragonal symmetry of the system. It is clear that it is only compatible with the $B_{2 g}$ nematic superconducting ground state, in which $\eta_{x}= \pm \eta_{y}$. Similarly, $\phi_{3}$ transforms as the $B_{1 g}$ irreducible representation, and is thus also a nematic vestigial order, compatible with the $B_{1 g}$ nematic superconducting state in which either $\eta_{x}=0$ or $\eta_{y}=0$. Finally, $\phi_{2}$ transforms as the $A_{2 g}$ irreducible representation, and thus breaks time-reversal symmetry, since $A_{2 g}$ corresponds to orbital angular momentum along the $z$ axis. Clearly, it is only compatible with the time-reversal symmetry-breaking superconducting ground state, in which $\eta_{x}= \pm i \eta_{y}$.

The coefficients of the terms that are quadratic in $\phi_{i}^{2}$ (with $i=1,2,3$ ), also called "masses" in field theory, determine which of the vestigial orders can appear. From Eqs. (6) and the Fierz identity we conclude that if $u-g<\min (0, w)$, the mass of the $\phi_{3}^{2}$ term is negative, and smaller than the masses of the $\phi_{1}^{2}$ and $\phi_{2}^{2}$ terms, indicating a tendency towards $B_{1 g}$ vestigial order. $u-g<\min (0, w)$ is also the condition that ensures that the ground state is the $B_{1 g}$ nematic superconducting state. Similar results hold in the other two regions of the parameter space $(u-g, w)$.

The key remaining question is whether the composite order parameter $\phi_{i}$ can condense even in the absence of superconducting order, i.e. whether the system can display a regime in which $\left\langle\boldsymbol{\eta}^{\dagger} \tau^{i} \boldsymbol{\eta}\right\rangle \neq 0$ but $\langle\boldsymbol{\eta}\rangle=0$. The $\phi_{i}$ are $Z_{2}$ (Ising-like) order parameters in this case, since they each transform according to one-dimensional irreducible representations, whereas $\boldsymbol{\eta}$ are complex $U(1)$ fields. Within mean-field, both the $Z_{2}$ and $U(1)$ symmetries are broken at the same temperature. However, once fluctuations are included, the natural result is that they are broken at two different temperatures or that a joint first order transition takes place. These two options are the generic behaviors of two order parameters that break different symmetries, whereas the simultaneous and second-order transition is only correct within a mean-field description. Since mean-field theory is appropriate for many superconductors, one expects quantitatively small effects. There are, however, a number of low-density and low-dimensional superconductors that are governed by sizable fluctuations of the superconducting order parameter and that are strong candidates for vestigial order. Examples are doped $\mathrm{Bi}_{2} \mathrm{Se}_{3}[30,31]$ and the half-Heusler systems $\mathrm{LuPtBi}$ and $\mathrm{YbPtBi}[33-36]$. In fact, the observed nematic order below $T_{c}$ in $\mathrm{Cu}$ - and $\mathrm{Sr}$-doped $\mathrm{Bi}_{2} \mathrm{Se}_{3}$ [37 42 strongly suggests a nematic phase above $T_{c}$ [3]. Due to the trigonal point group $D_{3 d}$ of this material, it follows that the vestigial nematic order parameter behaves like a three-state Potts model[43]. The cuprates are another class of materials where strong superconducting fluctuations are present. However, the gap function is $d_{x^{2}-y^{2}}$, which transforms as a one-dimensional irreducible representation of the $D_{4 h}$ group. Consequently, vestigial order related to superconductivity in the cuprates can only arise if there is additional translational symmetry breaking, as is the case for pair-density-wave states. Several recent works have focused on the issue of vestigial orders of the pair-density-waves, mostly in the context of the cuprates [6, 7, 44, 45].

\section{B. Model calculations}

Symmetry arguments can take us this far, but to proceed and determine whether the superconducting and vestigial orders are split, explicit calculations are necessary. Approaching the vestigial order from the melted ordered state, we parametrize the order parameter in terms of:

$$
\boldsymbol{\eta}(\mathbf{x})=\sqrt{n_{0}} e^{i \theta(\mathbf{x})}\left(\begin{array}{c}
\cos \alpha(\mathbf{x}) \\
e^{i \psi(\mathbf{x})} \sin \alpha(\mathbf{x})
\end{array}\right)
$$

with constant $n_{0}$. There are three coordinate-dependent phase variables: the global phase $\theta$, the relative phase $\psi$ between the two $p$-wave components $p_{x}$ and $p_{y}$, and the phase $\alpha$ that selects whether both components are simultaneously present. In each of the three vestigial phases, $\left\langle e^{i \theta(\mathbf{x})}\right\rangle=0$, implying that the system has no superconducting order. Moreover, either $\alpha$ or $\psi$ can acquire two values in a given vestigial phase, highlighting the Ising character of the composite order parameters (see Fig. 2p)

For concreteness, let us consider a two-dimensional system with $u-g>w>0$, which corresponds to the mean-field ground state $\boldsymbol{\eta}_{A_{2 g}} \propto(1, \pm i)$. The effective action $S \equiv F / T$, where $F$ is the total free energy has two contributions: the gradient term (we neglect here the coupling to the electromagnetic field)

$$
S_{\text {grad }}=\frac{1}{2 T} \int d^{2} x\left\{\left(\partial_{\mu} \theta\right)^{2}+\left(\partial_{\mu} \alpha\right)^{2}+\sin ^{2} \alpha\left(\partial_{\mu} \psi\right)^{2}+2 \sin ^{2} \alpha \partial_{\mu} \psi \partial_{\mu} \theta\right\}
$$

with some dimensionless temperature $T$, and the potential term

$$
S_{\text {pot }}=-\frac{\Delta}{a^{2} T} \int d^{2} x \sin ^{2}(2 \alpha) \sin ^{2} \psi,
$$


where $\Delta=w n_{0}^{2} a^{2}$ is a dimensionless constant with $w>0$ from Eq.(5). This action can be analyzed using renormalization-group techniques (for a related problem, see Ref. 46]). The key result is the onset of time-reversal symmetry (TRS) breaking at a temperature $T_{0} \sim 2 \pi / \log \left(\Delta^{-1}\right)$. For $T>T_{0}$, the gradient term dominates the renormalization-group flow, and the system behaves similarly to Heisenberg $O(3)$ spins. In this regime, the superconducting correlation length follows the usual behavior of the non-linear sigma model with spin correlation length $\xi\left(T>T_{0}\right)=a e^{\pi / T}$

Below $T_{0}$, the potential term starts to dominate. Because $\Delta>0$ increases under the renormalization group flow, the effect of this term is to lock the variables $\alpha$ and $\psi$ in order to minimize the energy, i.e. $\alpha=\frac{\pi}{4}$ and $\psi=\frac{\pi}{2}$ or $\psi=\frac{3 \pi}{2}$. As a result, the order parameter is that of a $p_{x} \pm i p_{y}$ superconductor with a fluctuating phase:

$$
\boldsymbol{\eta}(x)=\sqrt{\frac{n_{0}}{2}} e^{i \theta(x)}\left(\begin{array}{c}
1 \\
\pm i
\end{array}\right)
$$

Now the only relevant variable is the overall superconducting phase, such that the gradient term becomes

$$
S_{\text {grad }} \rightarrow \frac{1}{2 T} \int d^{2} x\left(\partial_{\mu} \theta\right)^{2}
$$

which is the same action as the usual XY-model. As a result, the system becomes governed by the BerezinskiiKosterlitz-Thouless (BKT) behavior of the XY-model with the key difference that the size of the vortex core is $\xi\left(T_{0}\right) \approx \frac{a}{\sqrt{\Delta}}$

Because $\xi\left(T_{\mathrm{BKT}}\right) \rightarrow \infty$, the BKT transition temperature $T_{\mathrm{BKT}}$ is clearly below $T_{0}$, even though we find, following Ref. 46, that both temperatures are parametrically of the same order. To unveil the meaning of the temperature $T_{0}$, we note that the potential term can be alternatively expressed in terms of the vestigial $A_{2 g}$ order parameter $\phi_{2}$

$$
S_{\text {pot }}=-\frac{\Delta}{a^{2} T} \int d^{2} x\left(\frac{\phi_{2}}{n_{0}}\right)^{2}
$$

As the correlation length increases, regions of typical size $\xi$ essentially share the same value of the Ising variable $\phi_{2} / n_{0} \approx \pm 1$. For a two-dimensional system, this implies that a true Ising-like phase transition takes place when the correlation length becomes comparable to the Ising domain-wall thickness $a / \sqrt{\Delta}$ :

$$
\Delta \frac{\xi(T)^{2}}{a^{2}} \approx 1
$$

This immediately yields $T_{\mathrm{c}, \text { Ising }}=T_{0}$. Thus, $T_{0}>T_{\mathrm{BKT}}$ signals a true Ising-like phase transition to the vestigial state that breaks time-reversal symmetry, but does not display quasi-long-range superconducting order. This result agrees with analyses of related models that also find an Ising order onsetting above the BKT transition [47, 48.

One can also approach the vestigial phase if the system is not exactly two-dimensional, i.e. if true superconducting long-range order can take place. Generally, different techniques can be employed, such as the renormalization-group [15. 49, 50, self-consistent Gaussian approximation [51, and the saddle-point large- $N$ approximation [15. For the specific case of a $p$-wave superconductor, the self-consistent Gaussian approximation was employed in Ref. [52. Here, we will focus on the large- $N$ approach: in this method, one starts with the free energy (6), complemented by the standard gradient terms, and decouples the quartic coefficients (quadratic in the bilinears) using HubbardStratonovich transformations. In the parameter regime relevant for $A_{2 g}$ superconducting order, $u-g>w>0$, it is sufficient to keep only the fields corresponding to the $\phi_{0}$ and $\phi_{2}$ bilinears, obtaining the action

$$
S=\int_{k} \boldsymbol{\eta}_{k}^{\dagger}\left[\left(r+\phi_{0}+k^{2}\right) \boldsymbol{\tau}_{0}+\phi_{2} \boldsymbol{\tau}_{y}\right] \boldsymbol{\eta}_{k}+\frac{\phi_{2}^{2}}{4 w}-\frac{\phi_{0}^{2}}{4(u+g+w)}
$$

In the disordered state, the fields $\boldsymbol{\eta}$ are fluctuating and can be integrated out exactly, resulting in an action that depends only on $\phi_{0}$ and $\phi_{2}$. The equation of state for $\phi_{2}$ can then be obtained using a saddle-point approximation, which is formally exact in the limit where the number $N$ of components of $\boldsymbol{\eta}$ is $N \rightarrow \infty$. To linear order in the vestigial order parameter $\phi_{2}$, one obtains:

$$
\frac{\phi_{2}}{w}=A \xi^{4-d} \phi_{2}
$$


where $A$ is some constant, $\xi(T)$ is the temperature-dependent correlation length, and $d>2$ is the dimensionality of the system. This equation allows a non-zero $\phi_{2}$ value at the critical temperature $T_{0}$ where $\xi\left(T_{0}\right)=(A w)^{-\frac{1}{4-d}}$, which takes place before the temperature $T_{c}$ in which long-range superconductivity appears, since $\xi\left(T_{c}\right) \rightarrow \infty$. For $d=2$ we recover the previous result for the correlation length at the vestigial transition. The only way to avoid vestigial order at a separate transition temperature $T_{0}>T_{c}$ is via a simultaneous first-order transition, since in this case $\xi\left(T_{c}\right)$ no longer diverges. This is the generic behavior that occurs in isotropic, three-dimensional systems [15. The split second-order transition in low-dimensional and anisotropic three-dimensional systems is a consequence of the enhanced role of fluctuations of the primary order parameter 53 . The quantum dynamics near $T=0$, however, may place the system closer to its upper critical dimension, thus reducing the impact of fluctuations and favoring a single first-order quantum transition [54] (see Figs. 17a and b).

These analyses reveal that there can be no single second-order phase transition into a multi-component superconductor: either there are two separate transitions or a single first-order transition. This simple yet robust result has important implications for the interpretation of experimental data on material candidates for $p$-wave superconductivity (see also Ref. [52]). The same behavior holds also for any superconducting state with a multi-component order parameter that transforms according to any of the 32 point groups of three-dimensional crystalline systems. Specifically, vestigial orders originating from superconductivity are possible for the 15 point groups with higher-dimensional irreducible representations, i.e. for all the cubic groups $T, T_{h}, T_{d}, O$, and $O_{h}$, the tetragonal groups $C_{4 v}, D_{2 d}, D_{4}$, and $D_{4 h}$, the hexagonal groups $C_{6 v}, D_{3 h}$, and $D_{6 h}$, and the trigonal groups $C_{3 v}, D_{3 d}$, and $D_{3}$. On the other hand, no vestigial order of translationally-invariant superconducting states occurs in an orthorhombic, monoclinic, or triclinic system.

\section{VESTIGIAL ORDER FROM DENSITY-WAVES IN THE SQUARE LATTICE}

We now proceed to apply the formalism developed above to classify possible vestigial orders arising from densitywaves on the square lattice. We start with the richer case of spin density-waves. As explained, the ground state must be degenerate in order for non-trivial composite operators to emerge. The standard Néel-like order, with wave-vector $\mathbf{Q}=(\pi, \pi)$, does not support vestigial orders that break the point group symmetry of the lattice. The simplest non-trivial case is then that of two degenerate magnetic ground states that are related by a symmetry of the lattice, corresponding to two ordering vectors $\mathbf{Q}_{1}=(\pi, 0)$ and $\mathbf{Q}_{2}=(0, \pi)$. The local spin can then be written as

$$
\mathbf{S}(\mathbf{r})=\mathbf{m}_{1} \cos \left(\mathbf{Q}_{1} \cdot \mathbf{r}\right)+\mathbf{m}_{2} \cos \left(\mathbf{Q}_{2} \cdot \mathbf{r}\right)
$$

where $\mathbf{m}_{a}$ are the real vector order parameters associated with $\mathbf{Q}_{a}$, where $a=1,2$. In the square lattice, there are three possible magnetic ground states [55, 56, illustrated in Fig. 3 a $C_{2}$-symmetric single- $\mathbf{Q}$ spin density-wave, corresponding to only one $\mathbf{m}_{a}$ being non-zero; a $C_{4}$-symmetric collinear double- $\mathbf{Q}$ spin density-wave, corresponding to $\mathbf{m}_{1} \| \mathbf{m}_{2} \neq 0$; and a $C_{4}$-symmetric non-collinear double- $\mathbf{Q}$ spin density-wave, corresponding to $\mathbf{m}_{1} \perp \mathbf{m}_{2} \neq 0$.

This description has been widely employed to discuss nematicity and magnetism in iron-based materials [15, 55,60 . It arises from either a $J_{1}-J_{2}$ localized spin model or an itinerant microscopic model with partially nested Fermi pockets [15, 22, 23, 55. In what follows, we will not repeat arguments that were extensively presented elsewhere [25, 61], but instead give a symmetry-based analysis of the allowed vestigial states.

We start by writing down the symmetry group of the problem without spin-orbit interaction:

$$
\mathcal{G}=C_{4 v}^{\prime \prime \prime} \times S O(3)
$$

Here, $C_{4 v}^{\prime \prime \prime}$ is called the extended point group [62, 63]. It corresponds to the standard point group $C_{4 v}$ supplemented by three translations: $T_{1}=(1,0), T_{2}=(0,1)$, and $T_{3}=(1,1)$. It is convenient to consider this group because the density-wave order parameters break translational symmetry. We do not include inversion symmetry explicitly here. Because we have a vector order parameter, it transforms under the irreducible representation $\Gamma=E_{5 g} \otimes \Gamma^{S=1}$. Since $d_{E_{5 g}}=2$ and $d_{S}=2 S+1$, the dimensionality of the irreducible representation of the primary order parameter is $d_{\Gamma}=2 \times(2+1)=6$. As a result, the order parameter can be written as $\eta_{A}=\left(\mathbf{m}_{1}, \mathbf{m}_{2}\right)$, where the $\mathbf{m}_{a}$ are threecomponent vectors in spin space. Note that such a classification of the primary order parameters was done in Ref. [63], from which we borrow the group-theory notation. Here, our goal is to systematically discuss the possible vestigial orders.

The bilinear forms can be analyzed by using the following results

$$
E_{5} \otimes E_{5}=A_{1} \oplus B_{2}^{\prime} \oplus A_{2}^{\prime} \oplus B_{1}
$$


(a)
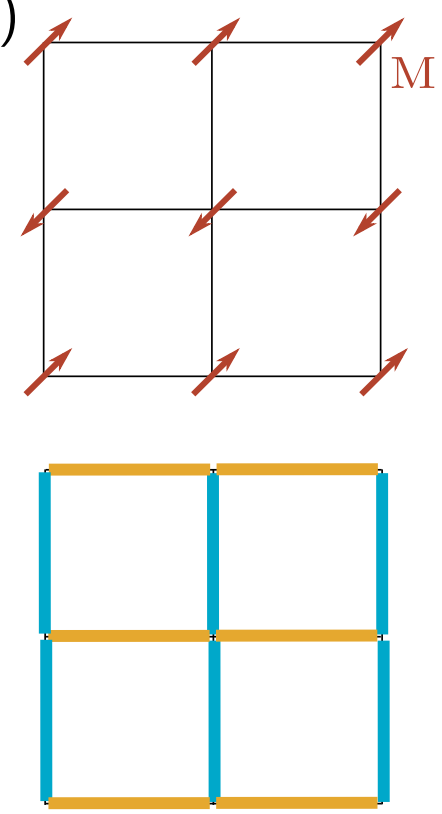

(b)

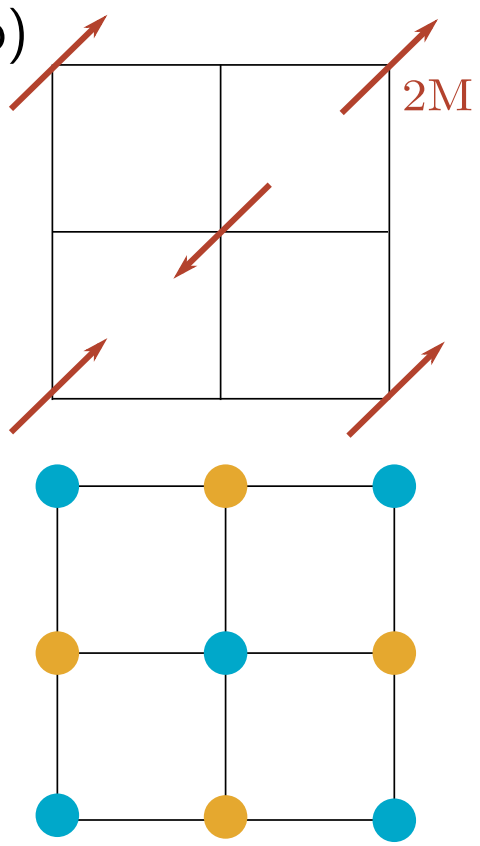

(c)
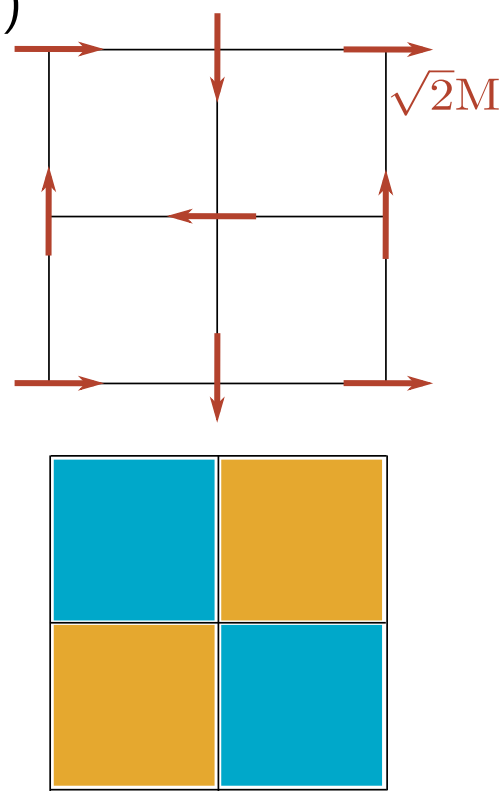

FIG. 3. Schematic representation of the three possible square-lattice spin density-wave ground states (upper panels) with ordering vectors $\mathbf{Q}_{1}=(\pi, 0)$ and $\mathbf{Q}_{2}=(0, \pi)$, and their corresponding vestigial phases (lower panels). Panel (a) refers to the single- $\mathbf{Q} C_{2}$-symmetric magnetic phase and its corresponding nematic vestigial phase, characterized by unequal bonds. Panel (b) shows the collinear double- $\mathbf{Q} C_{4}$-symmetric magnetic phase and its corresponding charge-ordered vestigial phase, characterized by unequal sites. Note that, in the magnetically ordered state, half of the sites have zero magnetization. Panel (c) illustrates the non-collinear double- $\mathbf{Q} C_{4}$-symmetric magnetic phase and its corresponding spin-current vestigial phase, characterized by unequal plaquettes. As indicated in the figure, the magnitudes of the local magnetization are different in each ordered state.

and

$$
\Gamma^{S *} \otimes \Gamma^{S}=\bigoplus_{j=0}^{2 S} \Gamma^{j}
$$

The primes in $A_{2}^{\prime}$ and $B_{2}^{\prime}$ indicate that translational symmetry is broken by $T_{3}=(1,1)$. We thus obtain:

$$
\Gamma^{*} \otimes \Gamma=\left(A_{1} \oplus B_{2}^{\prime} \oplus A_{2}^{\prime} \oplus B_{1}\right) \otimes\left(\Gamma^{0} \oplus \Gamma^{1} \oplus \Gamma^{2}\right)
$$

The index of a irreducible representation of the product, $m=(r, j)$, is then a combination of the four spatial irreducible representations $r=(0,1,2,3)=\left(A_{1}, B_{2}^{\prime}, A_{2}^{\prime}, B_{1}\right)$ and the spin $j$. As a result, the possible composite operators are written as:

$$
\phi_{m \equiv(r, j)}^{\mu}=\sum_{A, B} \eta_{A} \Lambda_{A, B}^{m, \mu} \eta_{B}
$$

with matrices

$$
\Lambda_{A, B}^{m \equiv(r, j), \mu}=\tau_{a b}^{r} \lambda_{\alpha \beta}^{j, \mu}
$$

These matrices transform according to one of the irreducible representations $\Gamma^{m}$ contained in the product $\Gamma^{*} \otimes \Gamma$. The number of matrices is given by the dimensionality $d_{m}$ of $\Gamma^{m}$, so that the index $\mu=1, \ldots, d_{m}$. The indices $A=(a, \alpha), B=(b, \beta)$ combine point and spin group indices, such that $\eta_{A} \equiv m_{a}^{\alpha}$. The $\tau_{a b}^{r}$ are the unit matrix $\tau^{0}$ and the three Pauli matrices $\tau^{r}$. The $3 \times 3$ matrices $\lambda^{j}$ for $j=0,1$, and 2 act in spin space and are given as follows: For $j=0$ we have $\lambda_{\alpha \beta}^{0,0}=\delta_{\alpha \beta}$, and the composite order parameter can be expressed as a scalar $\phi_{(r, j=0)}$. For $j=1$ we have 
three matrices $\lambda_{\alpha \beta}^{1, \mu}=i \epsilon_{\alpha \beta \mu}$ corresponding to the three anti-symmetric Gell-Mann matrices. Thus, we can express the composite order parameter as a vector $\phi_{(r, j=1)}$. Finally for $j=2$, we use the five symmetric Gell-Mann matrices. They can be labelled by a double index $\left(\mu, \mu^{\prime}\right)$ of a symmetric tensor, where $\mu$ and $\mu^{\prime}$ take three values each:

$$
\lambda_{\alpha \beta}^{2,\left(\mu, \mu^{\prime}\right)}=\frac{1}{2}\left(\delta_{\alpha \mu} \delta_{\beta \mu^{\prime}}+\delta_{\alpha \mu^{\prime}} \delta_{\beta \mu}\right)-\frac{1}{3} \delta_{\alpha \beta} \delta_{\mu \mu^{\prime}}
$$

In this case, the vestigial order parameter is a second-rank tensor $\phi_{(r, j=2)}^{\mu \mu^{\prime}}$. This exhausts all $3 \times 3$ matrices, which is what we expect for an order parameter that transforms as $S=1$.

We first consider $j=0$. There are three possible non-vanishing scalar bilinears

$$
\begin{aligned}
& \phi_{(0,0)}=\mathbf{m}_{1} \cdot \mathbf{m}_{1}+\mathbf{m}_{2} \cdot \mathbf{m}_{2} \\
& \phi_{(1,0)}=2 \mathbf{m}_{1} \cdot \mathbf{m}_{2} \\
& \phi_{(3,0)}=\mathbf{m}_{1} \cdot \mathbf{m}_{1}-\mathbf{m}_{2} \cdot \mathbf{m}_{2},
\end{aligned}
$$

Note that $\phi_{(2,0)}$ vanishes, since the $\mathbf{m}_{i}$ are real vectors. While $\phi_{(0,0)}$ transforms trivially $\left(A_{1}\right.$ representation), we obtain two vestigial order parameters that break spatial symmetries, without breaking spin-space symmetries. $\phi_{(3,0)}$, which transforms as $B_{1}$, is an Ising-nematic order parameter, which is frequently observed in iron-based systems (see Fig. 3 ) $)$. It is the vestigial phase of the single- $\mathbf{Q}$ magnetic ground state. $\phi_{(1,0)}$, which transforms as $B_{2}^{\prime}$, corresponds to a scalar that breaks translational symmetry (with ordering vector $\mathbf{Q}_{1}+\mathbf{Q}_{2}=(\pi, \pi)$ ), while preserving the tetragonal symmetry of the lattice (see Fig. 3p). It thus corresponds to a checkerboard charge order, and is the vestigial phase of the $C_{4}$-symmetric collinear double-Q magnetic state observed in several iron-based systems 64 71. Interestingly, in the phase diagrams of these compounds, the single- $\mathbf{Q}$ phase undergoes a transition to the double- $\mathbf{Q}$ phase as function of doping. A little explored problem is the interplay between the two corresponding vestigial orders in this case where the primary order itself changes (see Fig. 11).

For $j=1$, the only non-zero vestigial order is the vector composite order parameter

$$
\phi_{(2,1)}=2 \mathbf{m}_{1} \times \mathbf{m}_{2} .
$$

It corresponds to a vector chirality, which is manifested as spin current loops that are staggered between different plaquettes, forming an imaginary spin density-wave with ordering vector $\mathbf{Q}_{1}+\mathbf{Q}_{2}=(\pi, \pi)$ (see Fig. 35). It is the vestigial phase of the $C_{4}$-symmetric non-collinear double- $\mathbf{Q}$ magnetic state observed recently in doped $\mathrm{CaKFe}_{4} \mathrm{As}_{4} \mathbf{7 2}_{2}$. The three non-trivial states $\phi_{(1,0)}, \phi_{(3,0)}$, and $\phi_{(2,1)}$ were recently discussed in Ref. [56] and analyzed using a large- $N$ approximation. In a two-dimensional system, where long-range order of the primary order parameters is prohibited by the Hohenberg-Mermin-Wagner theorem, a vestigial phase having only the Ising-like order parameters $\phi_{(1,0)}$ or $\phi_{(3,0)}$ will take place. Note that the continuous composite order parameter $\phi_{(2,1)}$ cannot condense in a two-dimensional system; in Ref. [56, it was argued that a vestigial phase with $\phi_{(2,1)} \neq 0$ but $\mathbf{m}_{i}=0$ is possible in strongly anisotropic three-dimensional systems.

Finally, there is also the possibility for three more vestigial states with $j=2$ :

$$
\begin{aligned}
& \phi_{(0,2)}^{\mu \mu^{\prime}}=m_{1}^{\mu} m_{1}^{\mu^{\prime}}+m_{2}^{\mu} m_{2}^{\mu^{\prime}}-\frac{1}{3} \delta_{\alpha \beta}\left(\mathbf{m}_{1} \cdot \mathbf{m}_{1}+\mathbf{m}_{2} \cdot \mathbf{m}_{2}\right) \\
& \phi_{(1,2)}^{\mu \mu^{\prime}}=m_{1}^{\mu} m_{2}^{\mu^{\prime}}+m_{2}^{\mu} m_{1}^{\mu^{\prime}}-\frac{1}{3} \delta_{\mu \mu^{\prime}}\left(\mathbf{m}_{1} \cdot \mathbf{m}_{2}+\mathbf{m}_{2} \cdot \mathbf{m}_{2}\right) \\
& \phi_{(3,2)}^{\mu \mu^{\prime}}=m_{1}^{\mu} m_{1}^{\mu^{\prime}}-m_{2}^{\mu} m_{2}^{\mu^{\prime}}-\frac{1}{3} \delta_{\mu \mu^{\prime}}\left(\mathbf{m}_{1} \cdot \mathbf{m}_{1}-\mathbf{m}_{2} \cdot \mathbf{m}_{2}\right) .
\end{aligned}
$$

While $\phi_{(0,2)}^{\mu \mu^{\prime}}$ (with $\left.\mu, \mu^{\prime}=1,2,3\right)$ corresponds to pure spin-nematicity (i.e. nematic order in spin space, without affecting the lattice point group symmetry), the other two correspond to simultaneous rotational symmetry breaking in lattice and in spin space. Clearly, these order parameters mix if one includes spin-orbit interaction. However, it is still an interesting open question whether there are iron-based superconductors or other materials where these quadrupolar order parameters are the dominant vestigial order parameters.

The above analysis applies to any square-lattice system displaying density-waves with ordering vectors $\mathbf{Q}_{1}=(\pi, 0)$ and $\mathbf{Q}_{2}=(0, \pi)$. While we focused on the case of spin density-waves here, extension to the case of charge densitywaves is straightforward. In particular, in the case of (commensurate) charge density-waves, the possible vestigial orders are exactly the same as the $j=0$ composite order parameters discussed above. 

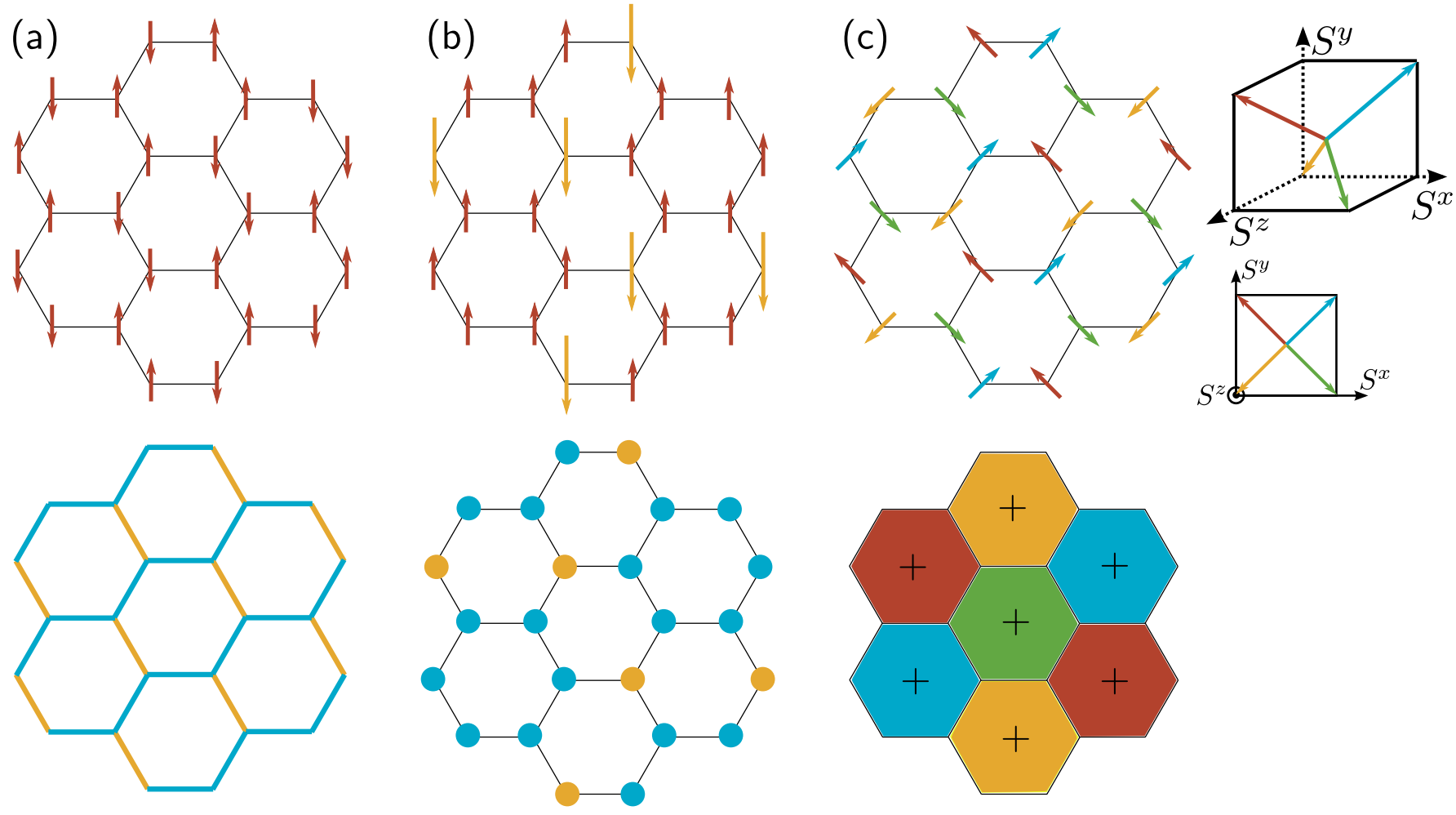

FIG. 4. Schematic representation of the three possible hexagonal-lattice spin density-wave ground states (upper panels) with ordering vectors $\mathbf{Q}_{1}=\frac{\pi}{\sqrt{3}}(\sqrt{3}, 1), \mathbf{Q}_{2}=\frac{\pi}{\sqrt{3}}(0,-2)$, and $\mathbf{Q}_{3}=\frac{\pi}{\sqrt{3}}(-\sqrt{3}, 1)$, and their corresponding vestigial phases (lower panels). Panel (a) refers to the single- $\mathbf{Q}$ magnetic phase and its corresponding nematic vestigial phase, characterized by unequal bonds. Panel (b) shows the collinear triple- $\mathbf{Q}$ magnetic phase and its corresponding charge-ordered vestigial phase, characterized by unequal sites. Note that the magnitude of the magnetization is three times larger in some sites (orange arrows) than in other sites (red arrows). Panel (c) illustrates the non-coplanar triple-Q magnetic phase, and its corresponding spin-current vestigial phase. In the magnetically ordered state, the local magnetization can point in one of the four directions shown in the upper inset; for simplicity, here we represent these four directions in the plane, as shown in the lower inset. The vestigial triple-Q spin-current phase is characterized by unequal plaquettes; specifically, there are four types of plaquettes corresponding to one of the four polarizations of the vector spin-chirality. Note that the scalar $\operatorname{spin}$-chirality $\mathbf{m}_{1} \cdot\left(\mathbf{m}_{2} \times \mathbf{m}_{3}\right)$ is positive and equal in each plaquette for the spin configuration drawn here.

\section{VESTIGIAL ORDER FROM DENSITY-WAVES IN THE HEXAGONAL LATTICE}

A similar analysis as the one outlined above can be performed for the case of density-waves in the hexagonal lattice. The new aspect of this problem is the existence of a triply-degenerate ground state, which allows us to discuss the case where the primary order parameter transforms as a three-dimensional irreducible representation. In this situation, non-trivial trilinear composite order parameters can exist, leading to an even richer phase diagram. We note that the classification of the primary order parameters for this situation was previously done in Refs. [63, 73]; we follow the notation of that paper to study the various composite orders.

In the case of spin density-waves, the local spin is parametrized in terms of three magnetic order parameters $\mathbf{m}_{a}$ associated with three wave-vectors related by $60^{\circ}$ rotations: $\mathbf{Q}_{1}=\frac{\pi}{\sqrt{3}}(\sqrt{3}, 1), \mathbf{Q}_{2}=\frac{\pi}{\sqrt{3}}(0,-2)$, and $\mathbf{Q}_{3}=$ $\frac{\pi}{\sqrt{3}}(-\sqrt{3}, 1)$, such that $\mathbf{Q}_{1}+\mathbf{Q}_{2}+\mathbf{Q}_{3}=0$ :

$$
\mathbf{S}(\mathbf{r})=\sum_{a=1,2,3} \mathbf{m}_{a} \cos \left(\mathbf{Q}_{a} \cdot \mathbf{r}\right)
$$

The three possible magnetic ground states, illustrated in Fig. 4, correspond to [74]: (i) a single-Q spin density-wave phase, in which only one of the $\mathbf{m}_{a}$ order parameters is non-zero; (ii) a collinear triple- $\mathbf{Q}$ spin density-wave, in which all three magnetic order parameters are non-zero and parallel or anti-parallel to each other; (iii) a non-coplanar triple- $\mathbf{Q}$ spin density-wave, in which again all three magnetic order parameters are non-zero and perpendicular to each other. 
Such a description has been employed to study the magnetic properties of graphene doped to its van-Hove singularity point and also of doped cobaltates [74 78]. More recently, it has been applied to $\mathrm{SrTiO}_{3}$ thin films grown along the (111) orientation [79]. It can be derived from an itinerant microscopic model with a nearly-nested Fermi surface [74].

The relevant group of the primary order parameter is given, in the absence of spin-orbit coupling, by

$$
\mathcal{G}=C_{6 v}^{\prime \prime \prime} \times S O(3)
$$

The extended point group $C_{6 v}^{\prime \prime \prime}$ corresponds to the point group $C_{6 v}$ supplemented by three translations $T_{1}=\frac{1}{2}(1, \sqrt{3})$, $T_{2}=\frac{1}{2}(1,-\sqrt{3})$, and $T_{3}=(1,0)$. The primary order parameters $\mathbf{m}_{a}$ transform according to the irreducible representation $\Gamma=F_{1} \otimes \Gamma^{S=1}$. Since $F_{1}$ is a three-dimensional irreducible representation, we have a nine-dimensional primary order parameter $\eta_{A}=\left(\mathbf{m}_{1}, \mathbf{m}_{2}, \mathbf{m}_{3}\right)$. To proceed and form the bilinear forms, we use Eq. (21) to decompose the product $\Gamma^{S} \otimes \Gamma^{S}$ and also

$$
F_{1} \otimes F_{1}=A_{1} \oplus E_{2} \oplus F_{2} \oplus F_{1} .
$$

Note that the decomposition of $F_{1} \otimes F_{1}$ does not only yield one-dimensional (1D) irreducible representations. Instead, in addition to the trivial irreducible representation $A_{1}$, we obtain the two-dimensional irreducible representation $E_{2}$ (corresponding to the degeneracy between $d_{x y}$ and $d_{x^{2}-y^{2}}$ in the hexagonal lattice), and the three-dimensional irreducible representations $F_{1}$ and $F_{2}$, which correspond to orders that break translational symmetry according to the wave-vectors $\mathbf{Q}_{1}, \mathbf{Q}_{2}$, and $\mathbf{Q}_{3}$.

Similarly to the case of the square lattice, we introduce the index $m=(r, j)$ that is a combination of the spatial irreducible representations $r=(0,1,2,3)=\left(A_{1}, E_{2}, F_{2}, F_{1}\right)$ and the spin index $j$. The bilinears are once again given by

$$
\phi_{m \equiv(r, j)}^{\nu \mu}=\sum_{A, B} \eta_{A} \Lambda_{A, B}^{m, \nu \mu} \eta_{B}
$$

with $A=(a, \alpha), B=(b, \beta)$ and matrices:

$$
\Lambda_{A, B}^{m \equiv(r, j), \nu \mu}=\Gamma_{a b}^{r, \nu} \lambda_{\alpha \beta}^{j, \mu}
$$

The spin-space matrices $\lambda_{\alpha \beta}^{j, \mu}$ are the same as the ones presented in the previous section. As for the nine $3 \times 3$ matrices $\Gamma_{a b}^{r, \nu}$, they can be expressed in terms of the identity matrix $\Gamma_{a b}^{0,0}=\delta_{a b}$ and the eight Gell-Mann matrices. Denoting them by the usual notation $\lambda_{a b}^{l}$, with $l=1, \ldots, 8$, we separate the eight matrices into one doublet and two triplets according to: $\Gamma_{a b}^{1, \nu}=\left\{\lambda_{a b}^{3}, \lambda_{a b}^{8}\right\}, \Gamma_{a b}^{2, \nu}=\left\{\lambda_{a b}^{2}, \lambda_{a b}^{7}, \lambda_{a b}^{5}\right\}$, and $\Gamma_{a b}^{3, \nu}=\left\{\lambda_{a b}^{1}, \lambda_{a b}^{6}, \lambda_{a b}^{4}\right\}$. In what follows, we focus on scalar and vector bilinears; rank-2 tensor bilinears can be obtained in the same way as in the previous section in a straightforward way.

For $j=0$, the bilinears are scalars and given by $\phi_{(r, 0)}^{\nu}=\sum_{a, b=1}^{3}\left(\mathbf{m}_{a} \cdot \mathbf{m}_{b}\right) \Gamma_{a b}^{r, \nu}$. We find six non-zero possible bilinears:

$$
\begin{aligned}
& \phi_{(0,0)}=m_{1}^{2}+m_{2}^{2}+m_{3}^{2} \\
& \phi_{(1,0)}^{\nu}=\left\{m_{1}^{2}-m_{2}^{2}, \frac{1}{\sqrt{3}}\left(m_{1}^{2}+m_{2}^{2}-2 m_{3}^{2}\right)\right\} \\
& \phi_{(3,0)}^{\nu}=2\left\{\mathbf{m}_{1} \cdot \mathbf{m}_{2}, \mathbf{m}_{2} \cdot \mathbf{m}_{3}, \mathbf{m}_{1} \cdot \mathbf{m}_{3}\right\}
\end{aligned}
$$

Note that $\phi_{(0,0)}$ transforms trivially as $A_{1}$ and thus cannot form a vestigial order. The two order parameters of $\phi_{(1,0)}^{\nu}$ transform non-trivially as the two-dimensional irreducible representation $E_{2}$ and correspond to nematic orders with $d_{x^{2}-y^{2}}$ and $d_{x y}$ form factors, respectively. These bilinears allow for a vestigial nematic state that lowers the point-group symmetry without breaking translational symmetry. They are the vestigial phase of the single-Q spin density-wave (see Fig. 4a).

The three order parameters of $\phi_{(3,0)}^{\nu}$ transform non-trivially as the three-dimensional irreducible representation $F_{1}$. They preserve the point-group symmetry of the lattice but break translational symmetry. Thus, they correspond to charge density-waves with ordering vectors $\mathbf{Q}_{3}(\nu=1), \mathbf{Q}_{1}(\nu=2)$, and $\mathbf{Q}_{2}(\nu=3)$, which are vestigial orders of the collinear triple-Q spin density-wave (see Fig. 4b). As shown in Ref. 77, the transition to the vestigial phase belongs 
to the same universality class of the 4 -state Potts model, corresponding to $\phi_{(3,0)}^{\nu}= \pm 1$ subject to the constraint $\prod_{\nu=1}^{3} \operatorname{sign}\left[\phi_{(3,0)}^{\nu}\right]= \pm 1$. Finally, the composite order parameters $\phi_{(2,0)}^{\nu}$ vanish as the three corresponding Gell-Mann matrices are purely imaginary, but $m_{a}^{\alpha}$ are real.

For $j=1$, we obtain vector bilinears according to $\phi_{(r, 1)}^{\nu}=\sum_{a, b}\left(\mathbf{m}_{a} \times \mathbf{m}_{b}\right) \Gamma_{a b}^{r, \nu}$. There are three non-zero such bilinears, which transform as the three-dimensional irreducible representation $F_{2}$ :

$$
\phi_{(2,1)}^{\nu}=2\left\{\mathbf{m}_{1} \times \mathbf{m}_{2}, \mathbf{m}_{2} \times \mathbf{m}_{3}, \mathbf{m}_{1} \times \mathbf{m}_{3}\right\}
$$

Each of them corresponds to spin-current density-waves (i.e. vector chirality) with ordering vectors $\mathbf{Q}_{3}(\nu=1)$, $\mathbf{Q}_{1}(\nu=2)$, and $\mathbf{Q}_{2}(\nu=3)$. The resulting vestigial order is thus the triple- $\mathbf{Q}$ spin-current order shown in Fig. 4 . This is the vestigial phase of the non-coplanar triple- $\mathbf{Q}$ spin density-wave.

Interestingly, because the primary order parameter transforms as a three-dimensional irreducible representation, it is possible to also construct trilinear forms $\psi^{m}=\sum_{A, B, C} \eta_{A} \eta_{B} \eta_{C} \Lambda_{A, B, C}^{m}$ that transform non-trivially. This can be formally done by combining vestigial order parameters $\phi_{(r, j)}^{\nu \mu}$ that transform as higher-dimensional irreducible representations and the primary order parameter. Among the bilinears presented in Eqs. (34) and (35), combining $\phi_{(2,1)}^{\nu}$, which transforms as $F_{2}$, with the primary order parameter $\eta_{A}$, which transforms as $F_{1}$, yields a composite trilinear scalar that transforms non-trivially according to the $A_{2}$ irreducible representation, since $F_{1} \otimes F_{2}=E_{2} \oplus F_{1} \oplus$ $F_{2} \oplus A_{2}$. In explicit form, the corresponding order parameter $\psi$ is given by:

$$
\psi=\mathbf{m}_{1} \cdot\left(\mathbf{m}_{2} \times \mathbf{m}_{3}\right) .
$$

We identify $\psi$ as the scalar chirality, an Ising-like, $\mathbf{Q}_{1}+\mathbf{Q}_{2}+\mathbf{Q}_{3}=0$, order parameter that breaks time-reversal symmetry [73, 80]. Similarly to the bilinear $\boldsymbol{\phi}_{(2,1)}^{\nu}$, it is also a vestigial phase of the non-coplanar triple- $\mathbf{Q}$ spin density-wave. This brings an interesting scenario, in which there are two different vestigial phases associated with the same primary order. While $\psi$ breaks a discrete symmetry, the vector chirality $\phi_{(2,1)}^{\nu}$ is a continuous order parameter. One thus expects the vestigial scalar chirality $\psi$ to order at a higher temperature than the vestigial spin-current density-waves $\phi_{(2,1)}^{\nu}$ in a sufficiently strongly anisotropic three-dimensional system (as schematically shown in Fig. 1.). A microscopic calculation of such a scenario remains to be seen.

We finish this section by discussing the case in which the primary order parameter is a charge density-wave. In this situation, one would expect vestigial orders corresponding to the $j=0$ composite order parameters of the spin density-wave case, namely, $\phi_{(1,0)}^{\nu}$ and $\phi_{(3,0)}^{\nu}$ in Eq. 34 . However, $\phi_{(3,0)}^{\nu}$ corresponds to charge density-waves with the same ordering vectors as the primary order parameters, and thus do not constitute a vestigial order. Moreover, $\phi_{(1,0)}^{\nu}$ cannot be realized, since it is not possible to form a single- $\mathbf{Q}$ charge-density wave. This follows from the fact that the trilinear $W_{1} W_{2} W_{3}$ (where $W_{a}$ correspond to the Ising-like charge density-wave order parameters) transforms trivially as $A_{1}$, implying that only triple- $\mathbf{Q}$ charge density-waves can be formed in the hexagonal lattice. As a result, even though the ground state is degenerate, in this particular case no vestigial order appears. Similar arguments imply that the nematic phase alone, which transforms as the two-dimensional irreducible representation $E_{2}$, Eq. (34), does not admit vestigial phases. This is because a cubic term appears in the free energy selecting the $d_{x^{2}-y^{2}}$ over the $d_{x y}$ nematic state 43 .

\section{OTHER EXAMPLES OF VESTIGIAL ORDER}

Besides the examples discussed above, there are several other systems that allow vestigial orders to appear. Here we discuss some of them, without the same level of details as in the previous sections. The example that we analyzed for the square lattice consisted of doubly-degenerate spin density-wave ordering vectors $(\pi, 0)$ and $(0, \pi)$. We mentioned that a non-degenerate ground state, such as the Néel order, which displays ordering vector $(\pi, \pi)$, does not allow vestigial orders that lower the point-group symmetry of the lattice. Yet, this does not imply that vestigial order is impossible for primary Néel order. Similarly to the composite order with $j=2$ discussed in Eq. 28), in the case of Néel order it is possible to form a rank-2 tensor bilinear analogous to $\phi_{(0,2)}^{\mu \mu^{\prime}}$ that breaks spin-rotational invariance while preserving the point group. This so-called spin-nematic phase [81] has been widely discussed in the context of spin-1 models. A candidate material for spin-nematic order is $\mathrm{NiGa}_{2} \mathrm{~S}_{4}[82$, which can be described by spin-1 Heisenberg spins on a triangular lattice.

Still focusing on the square lattice, it is also possible to have magnetic ground states with degeneracy higher than 2 , such as the fourfold-degenerate ordering vectors $\left(\frac{\pi}{2}, \frac{\pi}{2}\right),\left(-\frac{\pi}{2}, \frac{\pi}{2}\right),\left(\frac{\pi}{2},-\frac{\pi}{2}\right)$, and $\left(-\frac{\pi}{2},-\frac{\pi}{2}\right)$. Microscopically, such 

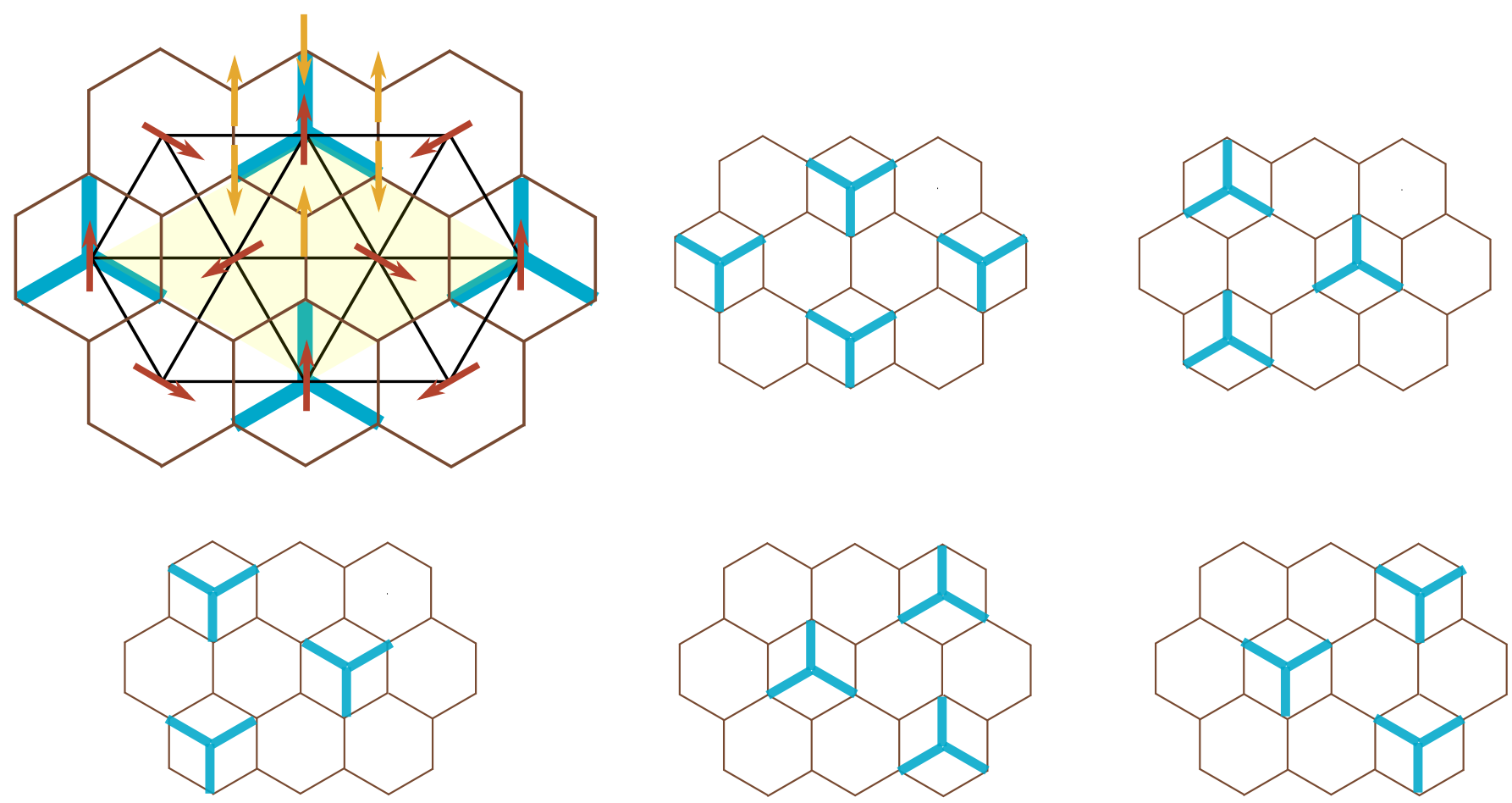

FIG. 5. Schematic representation of the magnetic ground state of the $J_{1}-J_{2}$ model on the windmill lattice, which is composed of interpenetrating triangular (black lines) and honeycomb (dark brown lines) sublattices. The spins order in a Néel pattern in the honeycomb sublattice and in a $120^{\circ}$ coplanar configuration in the triangular sublattice. The vestigial order is described in terms of a $\mathbb{Z}_{6}$ clock-model order parameter, and corresponds to different relative orientations between the spin orders of the two sublattices (blue bonds denote antiparallel spins).

states arise for instance in the $J_{1}-J_{2}-J_{3}$ Heisenberg model on the square lattice, with dominant $J_{3}$. Besides the socalled double-stripe magnetic ground states, plaquette ground states are also realized in this model [83. It was shown in Ref. 84 that the double-stripe magnetic state has two Ising-like vestigial orders, corresponding to a $B_{2 g}$ nematic order and a $(\pi, \pi)$ bond-order that breaks translational and reflection symmetries of the lattice. Experimentally, material candidates to exhibit these vestigial orders are the iron chalcogenide FeTe [85] 88, and the titanium-based oxypnictide $\mathrm{BaTi}_{2} \mathrm{Sb}_{2} \mathrm{O}$ [84. A full classification of all possible vestigial orders in this case is not yet available.

Another interesting case of multiple vestigial orders is that of the $J_{1}-J_{2}$ Heisenberg antiferromagnet on the windmill lattice, which consists of interpenetrating triangular and honeycomb sublattices 89 91. The $J_{1}-J_{2}$ windmill model is a straightforward, but non-trivial generalization of the corresponding $J_{1}-J_{2}$ square lattice model. It hosts a vestigial $Z_{6}$ clock order parameter instead of the Ising nematic $Z_{2}$ degree of freedom found on the square lattice. In both cases, vestigial order refers to a relative ordering of spins on the two sublattices. In the windmill case, it breaks translation symmetry by tripling the unit cell as well as a mirror symmetry that exchanges the $A$ and $B$ sites on the honeycomb sublattice (see Fig. 5p. Interestingly, due to the higher degree of degeneracy of the composite order parameter $\left(Z_{n}\right.$ with $n \geq 5$ ), vestigial long-range order develops via a two-step process consisting of two Kosterlitz-Thouless phase transitions that enclose an intermediate critical phase. In the critical phase, the correlations of the composite degrees of freedom decay algebraically with a temperature-dependent exponent $\eta(T)$ [91] (see Fig. 1f). Such a behavior is reminiscent of melting of two-dimensional solids, where (algebraic) translational order disappears via an intermediate hexatic phase 92 .

In all the density-wave examples discussed so far, only commensurate ordering vectors were considered. In the case of incommensurate wave-vectors, the primary order parameters become complex, which can lead to vestigial orders that break time-reversal symmetry. This was proposed for instance in Ref. 93] in the context of charge density-waves in the cuprates. Moreover, incommensurate spin density-wave with wave-vector $\mathbf{Q}$ naturally couples to incommensurate charge density-wave with wave-vector $2 \mathbf{Q}$, intrinsically coupling charge-driven and spin-driven nematicity. A full microscopic description of this peculiar case, which may be relevant for the cuprate $\operatorname{La}_{2-x} \mathrm{Sr}_{x} \mathrm{CuO}_{4}$, is still missing [51. The case of incommensurate charge density-wave also highlights the key role of disorder in vestigial phases: as shown in Ref. 94, in tw -dimensions, any amount of disorder kills incommensurate charge order at finite temperatures, while preserving its nematic vestigial phase. Overall, the impact of disorder on vestigial phases remains 
little explored, despite the ubiquity of disorder in realistic systems (see also Ref. [95]).

It is also important to emphasize that the existence of degenerate ground states is a necessary, but not sufficient condition for the appearance of vestigial orders. We already mentioned this feature in the case of charge densitywaves in the hexagonal lattice, which do not allow any vestigial phases. Another interesting example is the case of spin-dimers on a square lattice with nearest and next-nearest neighbor interactions and in the presence of an external magnetic field [96]. A closely related model has been employed to describe the unusual properties of $\mathrm{BaCuSi}_{2} \mathrm{O}_{6}$ in an external magnetic field 97 99. The model is equivalent to hard-core bosons on a square lattice that can undergo Bose-Einstein condensation at momenta $\mathbf{Q}_{1}=(\pi, 0)$ or $\mathbf{Q}_{2}=(0, \pi)$. As the chemical potential $\mu$ of these hard-core bosons is tuned from negative to positive, there is a quantum phase transition from a disordered state to a condensate with finite momentum. The model thus shares the same properties as the case of doubly-degenerate charge densitywaves on the square lattice discussed above. However, there is one important difference: at $T=0$ and at the quantum critical point $(\mu=0)$, the system is empty of bosons and fluctuations are thus irrelevant. As a result, there are no fluctuations to trigger a $T=0$ vestigial phase. Formally, this is manifested in the Ginzburg-Landau free-energy expansion by the vanishing of all the coefficients in front of the squared non-trivial bilinears (see for instance Eq. (6)). The resulting phase diagram has therefore a vestigial phase of the finite-momentum Bose-Einstein condensate only at finite temperatures, but a single second-order phase transition at $T=0$ (see Fig. 11.).

\section{CONCLUDING REMARKS}

The formalism developed here demonstrates that multi-component order parameters give rise to complex phase diagrams, providing an appealing framework to understand quantum materials that goes beyond the paradigm of competing phases. The degenerate nature of the ordered state - a necessary but not sufficient condition for the emergence of vestigial order - leads to the condensation of fluctuations at their own transition temperature, manifested by long-range order of composite operators. In this regard, composide order not only behaves as a vestige of the primary order, but it also affects the latter by lifting its degeneracy and thus relieving the frustration of the system. In situations where the primary order cannot establish long-range order, either due to strong thermal or quantum fluctuations or due to disorder, the vestigial order is the only sharp remanent of the primary order.

While symmetry arguments can efficiently be employed to classify which vestigial states are allowed in each case, they do not prove the actual existence of vestigial phases. Only via microscopic calculations of minimal models one can assess whether the vestigial and primary phase transitions take place at different temperatures or simultaneously as a first-order transition - in which case there is no vestigial order. Particularly near a quantum phase transition, the symmetry of the order parameter is not enough to determine the final behavior of the vestigial phase, as the dynamics of the primary order parameter plays an essential role. Theoretically, while mean-field calculations are incapable of capturing vestigial phases, a variety of controlled and uncontrolled analytical methods exist, such as the saddle-point large- $N$ approach [15, 94, the self-consistent Gaussian approximation [51, 52, and the renormalization-group approach [49, 50]. Numerically, vestigial order can be addressed straightforwardly by analyzing the statistical properties of the corresponding higher-order correlation functions. For example, the Ising nematic transition in the classical $J_{1}-J_{2}$ square lattice model has been directly observed using Monte-Carlo simulations [100, 101] (see Ref. [91] for a related study on the windmill lattice). An interesting further direction is to investigate vestigial order in low-dimensional quantum (spin) systems at zero temperature, where powerful numerical techniques are available[102].

The concept of vestigial order has thus the potential to be applied to a vast number of systems that have been partially explored or that even remain completely unexplored. An interesting issue that goes beyond broken-symmetry phases is whether topologically-driven orders may also support unusual vestigial states of matter [103].

\section{ACKNOWLEDGMENTS}

We thank C. Batista, E. Berg, P. Chandra, G. W. Chern, A. Chubukov, M. Christensen, P. Coleman, I. Eremin, R. Flint, E. Fradkin, M. Hecker, B. Jeevanesan, J. Kang, S. Kivelson, I. Mazin, J. Venderbos, and X. Wang for fruitful discussions and collaborations on topics reviewed in this work. R.M.F. is supported by the US Department of Energy, Office of Science, Basic Energy Sciences, under Award de-sc0012336. P.P.O. acknowledges support from Iowa State University Startup Funds. J.S. is supported by the Helmholtz Program Science and Technology of Nanosystems at 
the Karlsruhe Institute of Technology (KIT).

[1] Sachdev S, Demler E. 2004. Phys. Rev. B 69:144504

[2] Fernandes RM, Pratt DK, Tian W, Zarestky J, Kreyssig A, et al. 2010. Phys. Rev. B 81:140501

[3] Fernandes RM, Schmalian J. 2010. Phys. Rev. B 82:014521

[4] Fradkin E, Kivelson SA, Tranquada JM. 2015. Rev. Mod. Phys. 87:457-482

[5] Himeda A, Kato T, Ogata M. 2002. Phys. Rev. Lett. 88:117001

[6] Agterberg DF, Tsunetsugu H. 2008. Nature Physics 4:639-

[7] Berg E, Fradkin E, Kivelson SA. 2009. Nature Physics 5:830-

[8] Berg E, Fradkin E, Kivelson SA. 2009. Phys. Rev. B 79:064515

[9] Berg E, Fradkin E, Kivelson SA, Tranquada JM. 2009. New Journal of Physics 11:115004

[10] Loder F, Graser S, Kampf AP, Kopp T. 2011. Phys. Rev. Lett. 107:187001

[11] Chubukov AV, Pines D, Schmalian J. 2003. The physics of superconductors, chap. A Spin Fluctuation Model for d-Wave Superconductivity. Berlin: Springer, 495-590

[12] Lederer S, Schattner Y, Berg E, Kivelson SA. 2015. Phys. Rev. Lett. 114:097001

[13] Kivelson SA, Fradkin E, Emery VJ. 1998. Nature 393:550-

[14] Zaanen J, Nussinov Z, Mukhin S. 2004. Annals of Physics 310:181 - 260

[15] Fernandes RM, Chubukov AV, Knolle J, Eremin I, Schmalian J. 2012. Phys. Rev. B 85:024534

[16] Kosterlitz JM, Nelson DR, Fisher ME. 1976. Phys. Rev. B 13:412-432

[17] Aharony A. 2003. Journal of Statistical Physics 110:659-669

[18] Villain J. 1977. J. Phys. France 38:385

[19] Shender EF. 1982. Sov. Phys. JETP 56:178

[20] Henley CL. 1989. Phys. Rev. Lett. 62:2056-2059

[21] Chandra P, Coleman P, Larkin AI. 1990. Phys. Rev. Lett. 64:88-91

[22] Fang C, Yao H, Tsai WF, Hu J, Kivelson SA. 2008. Phys. Rev. B 77:224509

[23] Xu C, Müller M, Sachdev S. 2008. Phys. Rev. B 78:020501

[24] Fernandes RM, VanBebber LH, Bhattacharya S, Chandra P, Keppens V, et al. 2010. Phys. Rev. Lett. 105:157003

[25] Fernandes RM, Chubukov AV, Schmalian J. 2014. Nature Physics 10:97-

[26] Hergert W, Geilhufe M. 2018. Group theory in solid state physics and photonics,. Wiley VCH

[27] Dresselhaus M, Dresselhaus G, Jorio A. 2010. Group theory: Application to the physics of condensed matter. BerlinHeidelberg: Springer

[28] Mackenzie AP, Maeno Y. 2003. Rev. Mod. Phys. 75:657-712

[29] Kallin C. 2012. Rep. Prog. Phys. 75:042501

[30] Hor YS, Williams AJ, Checkelsky JG, Roushan P, Seo J, et al. 2010. Phys. Rev. Lett. 104:057001

[31] Kriener M, Segawa K, Ren Z, Sasaki S, Ando Y. 2011. Phys. Rev. Lett. 106:127004

[32] Sigrist M, Ueda K. 1991. Rev. Mod. Phys. 63:239-311

[33] Goll G, Marz M, Hamann A, Tomanic T, Grube K, et al. 2008. Physica B: Condensed Matter 403:1065 - 1067

[34] Butch NP, Syers P, Kirshenbaum K, Hope AP, Paglione J. 2011. Phys. Rev. B 84:220504

[35] Tafti FF, Fujii T, Juneau-Fecteau A, René de Cotret S, Doiron-Leyraud N, et al. 2013. Phys. Rev. B 87:184504

[36] Xu G, Wang W, Zhang X, Du Y, Liu E, et al. 2014. Scientific Reports 4:5709-

[37] Matano K, Kriener M, Segawa K, Ando Y, Zheng Gq. 2016. Nature Physics 12:852-

[38] Yonezawa S, Tajiri K, Nakata S, Nagai Y, Wang Z, et al. 2017. Nature Physics 13:123-

[39] Pan Y, Nikitin AM, Araizi GK, Huang YK, Matsushita Y, et al. 2016. Scientific Reports 6:28632-

[40] Du G, Li Y, Schneeloch J, Zhong RD, Gu G, et al. 2017. Science China Physics, Mechanics EJ Astronomy 60:037411

[41] Asaba T, Lawson BJ, Tinsman C, Chen L, Corbae P, et al. 2017. Phys. Rev. X 7:011009

[42] Shen J, He WY, Yuan NFQ, Huang Z, Cho Cw, et al. 2017. npj Quantum Materials 2:59-

[43] Hecker M, Schmalian J. 2017. ArXiv e-prints 1712.07523

[44] Wang Y, Agterberg DF, Chubukov A. 2015. Phys. Rev. Lett. 114:197001

[45] Cai RG, Li L, Wang YQ, Zaanen J. 2017. Phys. Rev. Lett. 119:181601

[46] Fellows JM, Carr ST, Hooley CA, Schmalian J. 2012. Phys. Rev. Lett. 109:155703

[47] Korshunov SE. 2002. Phys. Rev. Lett. 88:167007

[48] Hasenbusch M, Pelissetto A, Vicari E. 2005. Journal of Statistical Mechanics: Theory and Experiment 2005:P12002

[49] Qi Y, Xu C. 2009. Phys. Rev. B 80:094402

[50] Millis AJ. 2010. Phys. Rev. B 81:035117

[51] Nie L, Maharaj AV, Fradkin E, Kivelson SA. 2017. Phys. Rev. B 96:085142

[52] Fischer MH, Berg E. 2016. Phys. Rev. B 93:054501

[53] Karahasanovic U, Schmalian J. 2016. Phys. Rev. B 93:064520

[54] Fernandes RM, Maiti S, Wölfle P, Chubukov AV. 2013. Phys. Rev. Lett. 111:057001

[55] Lorenzana J, Seibold G, Ortix C, Grilli M. 2008. Phys. Rev. Lett. 101:186402

[56] Fernandes RM, Kivelson SA, Berg E. 2016. Phys. Rev. B 93:014511 
[57] Eremin I, Chubukov AV. 2010. Phys. Rev. B 81:024511

[58] Brydon PMR, Schmiedt J, Timm C. 2011. Phys. Rev. B 84:214510

[59] Giovannetti G, Ortix C, Marsman M, Capone M, van den Brink J, Lorenzana J. 2011. Nature Communications 2:398-

[60] Wang X, Kang J, Fernandes RM. 2015. Phys. Rev. B 91:024401

[61] Fernandes RM, Schmalian J. 2012. Superconductor Science and Technology 25:084005

[62] Serbyn M, Lee PA. 2013. Phys. Rev. B 87:174424

[63] Venderbos JWF. 2016. Phys. Rev. B 93:115107

[64] Kim MG, Kreyssig A, Thaler A, Pratt DK, Tian W, et al. 2010. Phys. Rev. B 82:220503

[65] Hassinger E, Gredat G, Valade F, de Cotret SR, Juneau-Fecteau A, et al. 2012. Phys. Rev. B 86:140502

[66] Avci S, Chmaissem O, Allred J, Rosenkranz S, Eremin I, et al. 2014. Nature Communications 5:3845-

[67] Wang L, Hardy F, Böhmer AE, Wolf T, Schweiss P, Meingast C. 2016. Phys. Rev. B 93:014514

[68] Bhmer AE, Hardy F, Wang L, Wolf T, Schweiss P, Meingast C. 2015. Nature Communications 6:7911-

[69] Allred JM, Avci S, Chung DY, Claus H, Khalyavin DD, et al. 2015. Phys. Rev. B 92:094515

[70] Hassinger E, Gredat G, Valade F, de Cotret SR, Cyr-Choinière O, et al. 2016. Phys. Rev. B 93:144401

[71] Allred JM, Taddei KM, Bugaris DE, Krogstad MJ, Lapidus SH, et al. 2016. Nature Physics 12:493-

[72] Meier WR, Ding QP, Kreyssig A, Budko SL, Sapkota A, et al. 2018. npj Quantum Materials 3:5-

[73] Venderbos JWF. 2016. Phys. Rev. B 93:115108

[74] Nandkishore R, Chern GW, Chubukov AV. 2012. Phys. Rev. Lett. 108:227204

[75] Li T. 2012. Europhys. Lett. 97:37001

[76] Wang WS, Xiang YY, Wang QH, Wang F, Yang F, Lee DH. 2012. Phys. Rev. B 85:035414

[77] Chern GW, Fernandes RM, Nandkishore R, Chubukov AV. 2012. Phys. Rev. B 86:115443

[78] Chern GW, Batista CD. 2012. Phys. Rev. Lett. 109:156801

[79] Boudjada N, Wachtel G, Paramekanti A. 2018. Phys. Rev. Lett. 120:086802

[80] Martin I, Batista CD. 2008. Phys. Rev. Lett. 101:156402

[81] Andreev AF, Grishchuk IA. 1984. Sov. Phys. JETP 60:267

[82] Nakatsuji A, Nambu Y, Tonomura H, Sakai O, Jonas S, et al. 2005. Science 309:1697

[83] Ducatman S, Perkins NB, Chubukov A. 2012. Phys. Rev. Lett. 109:157206

[84] Zhang G, Glasbrenner JK, Flint R, Mazin II, Fernandes RM. 2017. Phys. Rev. B 95:174402

[85] Li S, de la Cruz C, Huang Q, Chen Y, Lynn JW, et al. 2009. Phys. Rev. B 79:054503

[86] Bao W, Qiu Y, Huang Q, Green MA, Zajdel P, et al. 2009. Phys. Rev. Lett. 102:247001

[87] Xu C, Hu J. 2009. ArXiv e-prints 0903.4477

[88] Paul I, Cano A, Sengupta K. 2011. Phys. Rev. B 83:115109

[89] Orth PP, Chandra P, Coleman P, Schmalian J. 2012. Phys. Rev. Lett. 109:237205

[90] Orth PP, Chandra P, Coleman P, Schmalian J. 2014. Phys. Rev. B 89:094417

[91] Jeevanesan B, Chandra P, Coleman P, Orth PP. 2015. Phys. Rev. Lett. 115:177201

[92] Halperin BI, Nelson DR. 1978. Phys. Rev. Lett. 41:121-124

[93] Wang Y, Chubukov A. 2014. Phys. Rev. B 90:035149

[94] Nie L, Tarjus G, Kivelson SA. 2014. Proceedings of the National Academy of Sciences 111:7980-7985

[95] Cui T, Fernandes RM. 2018. ArXiv e-prints 1801.01988

[96] Loison D, Simon P. 2000. Phys. Rev. B 61:6114-6125

[97] Sebastian SE, Harrison N, Batista CD, Balicas L, Jaime M, et al. 2006. Nature 441:617-

[98] Batista CD, Schmalian J, Kawashima N, Sengupta P, Sebastian SE, et al. 2007. Phys. Rev. Lett. $98: 257201$

[99] Schmalian J, Batista CD. 2008. Phys. Rev. B 77:094406

[100] Weber C, Capriotti L, Misguich G, Becca F, Elhajal M, Mila F. 2003. Phys. Rev. Lett. $91: 177202$

[101] Kamiya Y, Kawashima N, Batista CD. 2011. Phys. Rev. B 84:214429

[102] Stoudenmire E, White SR. 2012. Annual Review of Condensed Matter Physics 3:111-128

[103] Chatterjee S, Sachdev S, Scheurer MS. 2017. Phys. Rev. Lett. 119:227002 